\title{
Nexus between directionality of terahertz waves and structural parameters in groove-patterned InAs
}

\author{
Jong-Hyuk Yim, ${ }^{1}$ Kyunggu Min, ${ }^{1}$ Hoonil Jeong, ${ }^{1}$ Jin-Dong Song, ${ }^{2}$ and Young-Dahl Jho ${ }^{1}$, \\ 1) School of Information and Communications, Gwangju Institute of Science and Technology, Gwangju 500-712, \\ South Korea \\ ${ }^{2)}$ Nano-Photonics Research Center, Korea Institute of Science and Technology, Seoul 136-791, \\ South Korea
}

(Dated: 25 August 2018)

We have performed terahertz $(\mathrm{THz})$-time domain spectroscopy in various geometries, for characterizing the directivity of $\mathrm{THz}$ waves emitted from groove-patterned InAs structures. First, we have identified two transient transport processes as underlying THz emission mechanisms in InAs epilayers with different thickness. The carrier drift around surface depletion region was predominant in thin sample group (10-70 nm) while the electronic diffusion was overriding the oppositely aligned drifting dipoles in thick sample group (370-900 $\mathrm{nm}$ ) as revealed via amplitude change and phase reversal. By a combined use of electron-beam lithography and inductively coupled plasma etching in $1 \mu \mathrm{m}$-thick InAs epilayers, we have further periodically fabricated either asymmetric V-groove patterns or symmetric parabolic apertures. The $\mathrm{THz}$ amplitude was enhanced, particularly along line-of-sight transmissive direction when the groove patterns act as microscale reflective mirrors periodically separated by a scale of diffusion length.

\section{INTRODUCTION}

Over the past two decades, there has been great progress in the generating and utilizing terahertz $(\mathrm{THz})$ waves for various applications, including imaging and communications ${ }^{1}$ Among various $\mathrm{THz}$ generation methods, ${ }_{2}^{2}$ semiconductor surface emitters combined with pulsed laser excitations has been one of the well-established methods, ${ }^{3,4}$ which are described by either non-linear process ${ }^{5}$ or surge current model ${ }^{6}$; photocarrier accelerations due to the surface field in wide bandgap semiconductors or dipole currents by the efficient separation between electrons and holes in narrow bandgap semiconductors (so-called, Photo-Dember effect).

Indium-based alloys are very attractive as contact-free $\mathrm{THz}$ emitters because of the large diffusion velocity difference between electrons and holes. Especially, InAs and InSb have the much higher electron mobilities (up to $30,000 \mathrm{~cm}^{2} / \mathrm{V} \cdot \mathrm{s}$ and $\left.76,000 \mathrm{~cm}^{2} / \mathrm{V} \cdot \mathrm{s}\right)$ than hole mobilities (up to $450 \mathrm{~cm}^{2} / \mathrm{V} \cdot \mathrm{s}$ and $850 \mathrm{~cm}^{2} / \mathrm{V} \cdot \mathrm{s}$ ) $?^{7}$ In the case of infrared pulse excitation $(\lambda \sim 800 \mathrm{~nm}$, the central wavelength of Ti:sapphire laser technology) on InAs and InSb, the absorption depth $(\sim 100 \mathrm{~nm})$ is much shorter than the diffusion scale $(\sim 1 \mu \mathrm{m})$ and the large excess energy $(>1 \mathrm{eV})$ leads to the even higher mobilities of photo-excited carriers $\stackrel{8}{-}$ In this context, InAs has been widely used as the most intense contactless $\mathrm{THz}$ emitter whereas InSb application has been limited probably due to the suppressed emission amplitude via the scattering of electrons into the $\mathrm{L}$ valley. 6

Although $\mathrm{THz}$ efficiency of InAs is appropriate for contact-free $\mathrm{THz}$ applications, directivity of $\mathrm{THz}$ waves was hardly controllable. In general, semiconductor-based

\footnotetext{
a) Electronic mail: jho@gist.ac.kr
}

THz time-domain spectroscopy (THz-TDS) under pulsed laser excitations are implemented based on many guiding components such as parabolic mirrors and lenses along reflective directions. Coordinating many optical components along the reflective directions leads to difficulty in controlling the direction of $\mathrm{THz}$ waves. Recently, THz studies based on diffusive semiconductors have been destined toward the easier availability and controllability of diffusions; $\mathrm{THz}$ microscopy system was instrumented with excellent spatial resolution using an optical fiber coupled with tilted InAs tips ${ }^{9}$ and $\mathrm{THz}$ amplitude enhancement was achieved by integrating periodic metal strip lines on $\operatorname{In}_{0.53} \mathrm{Ga}_{0.47}$ As layers for lateral diffusion currents: $\frac{10}{\underline{1}}$ Furthermore, increased $\mathrm{THz}$ magnitude was reported in magnetic fields up to $8 \mathrm{~T}$ at 170 $\mathrm{K}, \stackrel{11}{=}$ or in microstructured large-area photoconducting emitter with external electric field up to $120 \mathrm{kV} / \mathrm{cm}$ at room temperature $\underline{12}$ In this paper, we have implemented micro-scale groove patterns acting either as mirrors in IR range (in the case of $\mathrm{V}$-groove patterns) or as grids for lateral symmetry breaking (in the case of parabolic apertures), aiming for characterizing the corresponding directivity of $\mathrm{THz}$ waves as being measured in reflective, transmissive and lateral detection geometries.

\section{SAMPLES AND SCHEMES}

The conventional THz-TDS measurements 13 were performed under the illumination of a Ti:sapphire-based laser at $300 \mathrm{~K}$ whose pulse duration was $150 \mathrm{fs}$ pulse at the center wavelength of $800 \mathrm{~nm}$. The samples were coordinated with photo-conductive antennas (PCA) in various detection geometries for characterizing $\mathrm{THz}$ waves along different directions as cartooned in Fig. 1. The IR laser source was incident on the sample surface at $\theta=45^{\circ}$ in the reflective geometry, otherwise at $\theta=90^{\circ}$ focused onto 
(a)

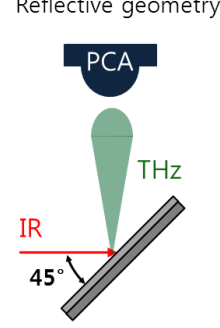

(b) Transmissive geometry

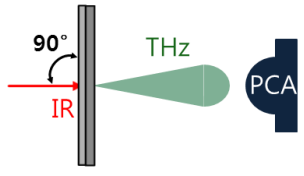

(c)

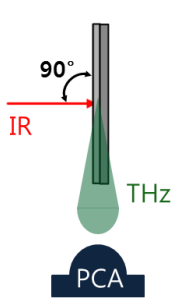

FIG. 1. Measurement schemes in (a) reflective (b) transmissive, and (c) lateral geometries.

a spot size of $800 \mu \mathrm{m}$. The pump beam supplies an excitation fluence of about $0.9 \mu \mathrm{J} / \mathrm{cm}^{2}$ which corresponds to the linear regime in (100)-grown InAs. $\stackrel{14}{ }$ In the case of reflective and transmissive geometries, $\mathrm{THz}$ waves from samples are guided via a pair of off-axis parabolic mirrors into PCA whose sensitivity was optimized at around $1 \mathrm{THz}$. On the other hand, in the lateral detection geometry, PCA was placed very close to the sample $(1 \mathrm{~mm}$ away from the sample edge) without guiding components.

The samples are classified into two categories; group A) bare InAs epilayers whose thickness was varied from $10 \mathrm{~nm}$ to $900 \mathrm{~nm}$, and group B) $1 \mu$ m-thick samples with groove-patterns around $1 \mu \mathrm{m}$. The detailed structures and relevant fabrication procedures are described in section III-A for group A and in in section III-B for group $\mathbf{B}$, respectively.

\section{RESULTS AND DISCUSSION}

\section{A. Determination of $\mathrm{THz}$ generation mechanism as a function of thickness}

The transient carrier transport mechanisms contribute to the emission of $\mathrm{THz}$ waves when surfaces of semiconductor materials are illuminated by laser pulses; drift currents by photo-carriers around the surface depletion regions could be biased either towards or against the substrate side, depending on the doping type, whereas the diffusion (via photo-Dember effect) occurs inherently toward the substrate side. As discussed earlier, InAs is one of the most efficient contactless $\mathrm{THz}$ emitters, whose generation mechanism has been associated with photoDember effect in very thick samples $(>100 \mu \mathrm{m})^{\frac{3}{3}}$. To examine the $\mathrm{THz}$ wave generation mechanisms in InAs layer whose thickness is comparable to or even smaller than the diffusion length scale, we have grown five unintentionally $n$-type doped InAs epilayers whose thickness was 10, 20,70, 370, and $900 \mathrm{~nm}$. To avoid the nonlinear effects, InAs was oriented along (100) direction, grown on GaAs substrate (thickness $\sim 300 \mu \mathrm{m}$ ) as shown in Fig. 2(a). To reduce the lattice mismatch and concomitant strain, $\mathrm{AlAs}_{0.32} \mathrm{Sb}_{0.68}$ layer (thickness $\sim 2.2 \mu \mathrm{m}$ ) was inserted between InAs and GaAs substrate. At $300 \mathrm{~K}$,

(a)
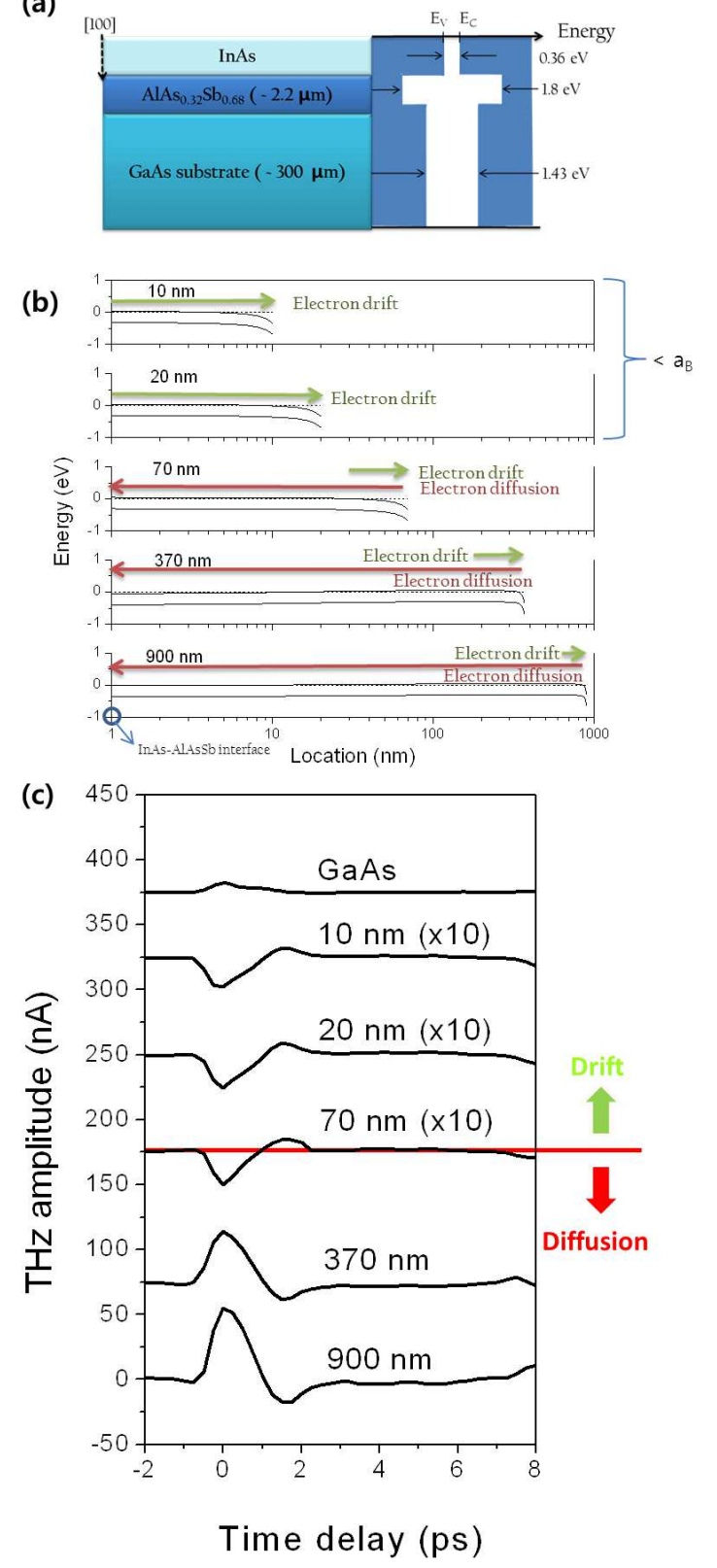

FIG. 2. (a) InAs epilayers grown on $\mathrm{AlAs}_{0.32} \mathrm{Sb}_{0.68}$ buffer layer and GaAs substrate, illustrated with the sketchy band gaps. (b) Energy band diagrams of InAs layers as a function of thickness. Green arrows and red arrows indicate electronic drift or diffusion field directions, respectively. (c) $\mathrm{THz}$ waveforms from InAs layers with different thicknesses, in comparison with GaAs substrate (from back side illumination of laser). Vertical arrows on the right-hand side divide $\mathrm{THz}$ emission regime, based either on the carrier drift motion (in thin sample group) or on the carrier diffusion (in thick sample group) with different phase.

bandgap of materials are roughly illustrated, in parallel with sample structures on the right-hand side of Fig. 2 (a). It is known that the optical rectification effect is 
minimized along (100) direction ${ }^{15}$, thus, the transport mechanisms are discussed from a perspective viewpoint of $\mathrm{THz}$ wave generation in this and following subsections.

In the energy band diagram of Fig. 2(b) as a function of sample thickness and $n$-type sheet carrier densities (measured elsewhere) ${ }^{16}$, we found that the electronic drift due to the tilted potentials at the surface was set to be opposite to the direction of carrier diffusion if available. In the quantum-confined scale of 10 and $20 \mathrm{~nm}$ (thin sample group) which is smaller than the exciton Bohr radius of $36 \mathrm{~nm}$ in a bulk InAs $\frac{17}{7}$, the diffusive features are mostly suppressed in contrast to the transient electron-hole separation due to the tilted potential shape around the surface. In $70 \mathrm{~nm}$-thick layer (thin sample group), the direction of transient drift currents are opposite to that of the partly allowed diffusion currents cancelling the net current flow. In thick sample group of $370 \mathrm{~nm}$ and $900 \mathrm{~nm}$, the carrier diffusion (mostly dominated by electrons) can occur throughout the InAs layer, whereas the oppositely aligned drifting electrons are spatially localized within the surface depletion layer of about $50 \mathrm{~nm}$.

Such thickness-dependent transport features are further confirmed by tracing the amplitudes and phases of $\mathrm{THz}$ waves which are associated with the transient gradient of dipole density, ambient electric field strength and dipole polarizations. Fig. 2(c) shows the THz-TDS results in reflection geometry as a function of InAs layer thickness. As a reference, GaAs substrate was illuminated from backward direction and the corresponding $\mathrm{THz}$ waves were displayed on top of Fig. 2(c). The THz amplitude showed rapid increment after $70 \mathrm{~nm}$. This amplitude variation in samples thicker than the absorption depth $(\sim 100 \mathrm{~nm})$ and exciton Bohr diameter is understood based on the efficient carrier diffusion and subsequent $\mathrm{THz}$ wave radiation. We further note that the opposite direction of electronic motions between the thin samples (dominated by the carrier drift) and thick sample group (dominated by the diffusion) led to the opposite phase of $\mathrm{THz}$ waves as indicated by the vertical arrows in Fig. 2(c). Intriguingly, $70 \mathrm{~nm}$-thick sample showed even smaller $\mathrm{THz}$ amplitude than thinner samples, which testifies to the barely initiated diffusions cancelling out the influence from electron drift along opposite direction. In this regard, in a thickness range larger than the 370 $\mathrm{nm}$, the underlying $\mathrm{THz}$ wave generation mechanisms is based on the electronic diffusion which will be further engineered for directional control of $\mathrm{THz}$ waves in the following section III-B.

\section{B. Directional $\mathrm{THz}$ emission modulated by groove-patterns}

InAs epitaxial layers (thickness $\sim 1 \mu \mathrm{m}$ ) were grown by molecular beam epitaxy along (100) direction on a GaAs substrate (thickness $\sim 500 \mu \mathrm{m}$ ) as shown in figure $3(\mathrm{a})$. InAs layers were $p$-type doped with a concentra- (a)

(b)
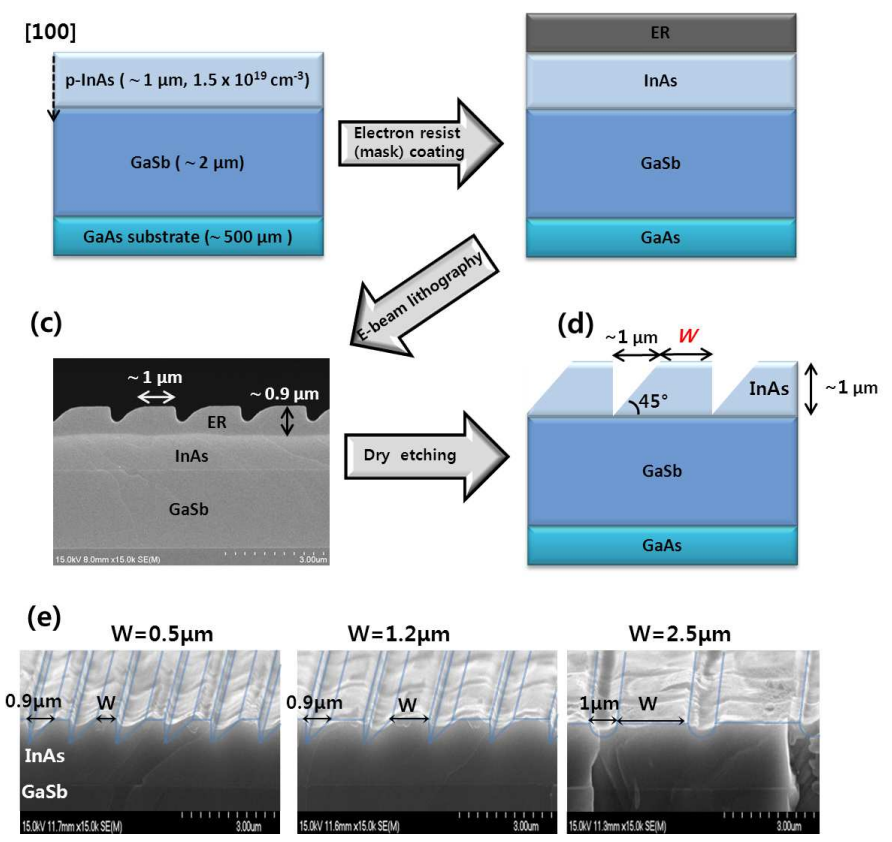

FIG. 3. Schematic fabrication processes for forming the groove patterns: (a) the bare InAs epilayer structure, (b) electron-resist (ER) layer, coated on InAs structure, (c) SEM image after E-beam writing and developing, and (d) finalized structure by dry etching process. (e) SEM images of the fabricated structures with different values of $\boldsymbol{W}(0.5 \mu \mathrm{m}$ and $1.2 \mu \mathrm{m}$ with trapezoidal patterns, and $2.5 \mu \mathrm{m}$ with parabolic patterns).

tion of $\sim 1.5 \times 10^{19} \mathrm{~cm}^{-3}$. A GaSb layer (thickness $\sim 2$ $\mu \mathrm{m})$ was inserted as a buffer layer to reduce the interfacial lattice mismatch. To fabricate the groove patterns, electron resist (ER) was coated on top of InAs layer (Fig. 3(b)). The electron beam writing was adapted for developing groove patterns on the ER using shotmodulation in electron-beam lithography, followed by dry etching process using inductively coupled plasma etcher with combined use of composite gas of $\mathrm{Cl}_{2}$ and $\mathrm{N}_{2}$ for forming groove patterns. Fig. 3(d) shows the proposed structure with a trapezoidal groove angle of $45^{\circ}$ with pattern width $(\boldsymbol{W})$. The pattern width $\boldsymbol{W}$ was varied from $0.5 \mu \mathrm{m}$ and $1.2 \mu \mathrm{m}$ to $2.5 \mu \mathrm{m}$ as shown in the SEM images of Fig. 3(e). It is notable that $\boldsymbol{W}$ was varied in a comparable scale to the electronic diffusion length in InAs $(\sim 1.3$ $\mu \mathrm{m}) \stackrel{18}{\underline{n}}$ The blue lines in Fig. 3(e) are guide to eyes for intended structures either with trapezoid-shaped grooves (in the case of $\boldsymbol{W}=0.5$ and $1.2 \mu \mathrm{m}$ ) or with parabolicaperture grooves (in the case of $\boldsymbol{W}=2.5 \mu$ ). During the fabrications, the edges of groove patterns were slightly deformed, identified as the deviation of SEM images from the blue lines in Fig. 3(e). The area of groove-patterned region was estimated to be about $5 \times 1 \mathrm{~mm}^{2}$.

Figure 4(a) shows a comparison between THz emission in reflective and in transmissive geometries in InAs bare 
samples as a reference. As expected, the signal along the transmissive direction in Fig. 4(a) was mostly suppressed since the diffusive dipoles are mostly aligned along the growth direction with relatively much weaker lateral components and the subsequent $\mathrm{THz}$ radiation patterns are perpendicular to transient currents. In addition, the $\mathrm{THz}$ waves generated within the InAs layer along the lateral direction are restrictedly coupled with the internal radiation cone along surface-normal direction due to the large mismatch in refractive index at the surface (the refractive index of $\sim 3.5$ for InAs compared to $\sim 1.0$ for free space) $\stackrel{19}{19}$ The small and slow oscillatory features after 10 ps could be ascribed to Fabry-Perot interference between air-InAs and air-GaAs interfaces.

In the case of groove-patterned structures, in clear contrast to the bare layer, we found that the $\mathrm{THz}$ signals were significantly enhanced along transmissive direction as shown in Fig. 4(b). The incident beam was normal to the surface and polarized linearly and orthogonally to the striped patterns. Due to the formation of diffusioninduced transient dipoles along the surface via the reflected IR pulses toward the lateral direction, the $\mathrm{THz}$ radiation patterns are now profiled toward the line-ofsight direction, manifested by the increased amplitude in Fig. 4(b). Particularly, a sample with $\boldsymbol{W}$ of $1.2 \mu \mathrm{m}$ turned out to be the most efficient which was in a similar scale to the diffusion length in InAs. ${ }^{18}$ The signals from a sample with $\boldsymbol{W}$ of $0.5 \mu \mathrm{m}$ was less enhanced in that the electrons can easily diffuse from one side to the other, thus, could be attracted to the rather stationary holes. In the case when $\boldsymbol{W}=2.5 \mu \mathrm{m}$, the patterns could break the lateral symmetry of diffusion but with limited lateral diffusion efficiency, considering the large laser spot size compared to $\boldsymbol{W}$. Some further arguments could be raised regarding the results in Fig. 4(b); 1) the FabryPerot interference was persistent in patterned structures, implying that the refractive index contrast at the interfaces was still persistent. Thus, the radiation outcoupling efficiency between the inside the materials and the free space seems not influenced by the groove patterns. 2) photocarriers generated in slope pyramid plane in the trapezoidal patterns could undergo the diffusion along the (110) direction with the much reduced carrier mobility and with heavier effective mass compared to those along (100) direction, which leads to the reduced photoDember field and the limited amplitude enhancement. 3) The not-perfectly defined faces of groove patterns could lead to the scattering loss of the incident IR photons. The pattern scale was much smaller than the $\mathrm{THz}$ wavelength, possibly triggering the Rayleigh scattering. Such arguments could be further discussed elsewhere based on spectrum-domain studies and angle-dependent amplitude analysis. Along the lateral direction (Fig. 4(c)), in clear contrast to transmissive direction, we could not observe the signal changes between patterned structures and the bare samples. The signals in general in Fig. 4(c) were much weaker than those in other geometries, possibly due to the slightly misaligned $\mathrm{THz}$ guiding optics,
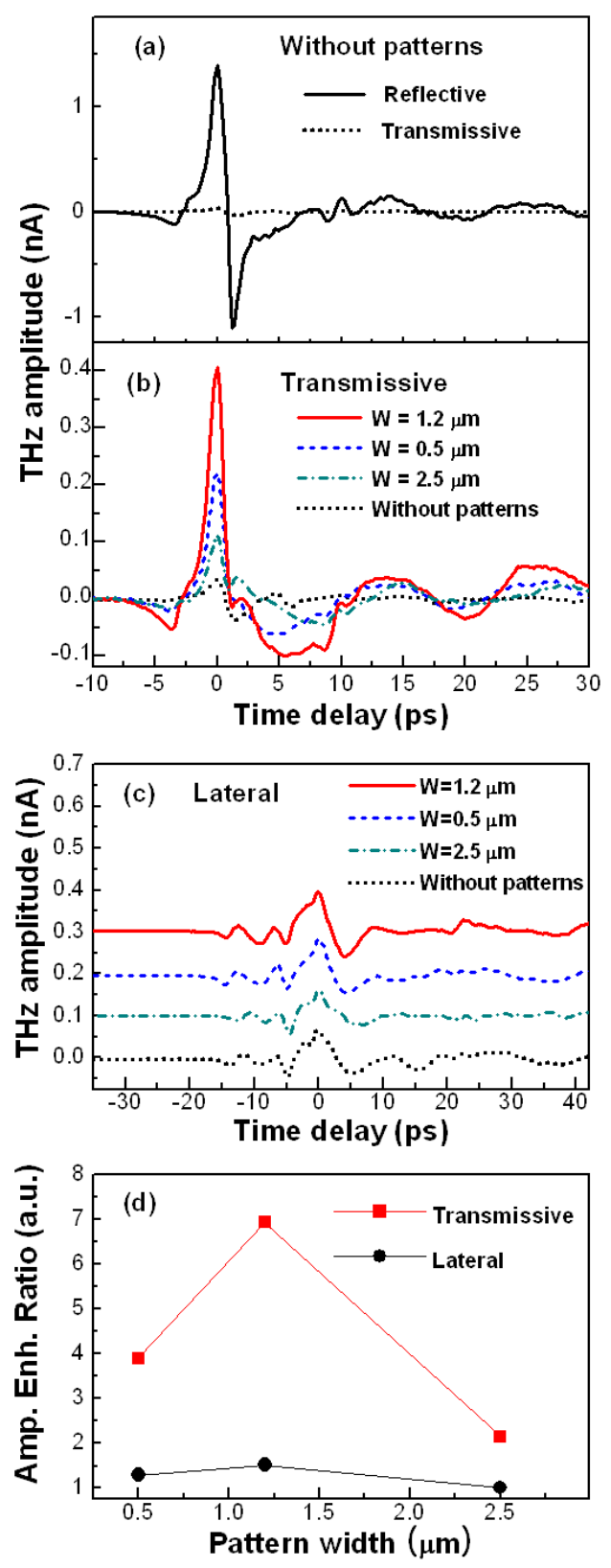

FIG. 4. Time-domain THz-TDS signals measured from (a) bare InAs layers in the reflective or transmissive geometries, (b) groove-patterned structures in comparison with the bare InAs layer (black-dotted line) in the transmissive geometry, and (c) groove-patterned structures in comparison with the bare InAs layer in the lateral geometry. (d) Amplitude enhancement ratio of $\mathrm{THz}$ waves along transmissive and lateral directions, normalized by amplitudes in bare InAs layer.

the scattering losses at the rough facets, and attenuation inside the medium. As a comparative purpose, we normalized $\mathrm{THz}$ signal amplitudes from the patterned structures either in the transmissvie or in lateral geometries by those from the bare InAs layer in Fig. $4(\mathrm{~d})$. $\mathrm{THz}$ wave amplitudes were increased significantly depending 
on the structural parameters of the periodic groove patterns, only along the line-of-sight trasmission direction. The highest amplitude enhancement ratio at $\boldsymbol{W}=1.2 \mu \mathrm{m}$ indicates that the optimized adjustment of $\boldsymbol{W}$ could be further obtained around the diffusion length.

\section{SUMMARY}

For a purpose of directional control of THz waves, 1 $\mu \mathrm{m}$-thick InAs epilayers were processed to have either 45 degree reflectors (with gap width $\boldsymbol{W}$ of 0.5 or 1.2 $\mu \mathrm{m})$ or parabolic apertures with $\boldsymbol{W}$ of $2.5 \mu \mathrm{m}$. The $\boldsymbol{W}$ and the sample thickness were designed to be similar to the diffusion length in which scale the $\mathrm{THz}$ emissions are predominantly generated by photo-Dember effect. We have performed $\mathrm{THz}-\mathrm{TDS}$ measurements excited with a pulsed IR source of Ti:sapphire laser at $300 \mathrm{~K}$. THzTDS resulted in a dramatic contrast among different emission directions. In patterned structures, specifically with mirror-like trapezoidal patterns, $\mathrm{THz}$ signals were enhanced along the transmissive direction whereas the lateral $\mathrm{THz}$ emission was not varied by the additional patterning. The enhancement was rather associated with the efficient lateral dipole formation, optimized when $\boldsymbol{W}$ was similar to the diffusion length.

\section{ACKNOWLEDGMENTS}

This work was supported by the Bio-Imaging Research Center at GIST and by the Basic Science Research Program through the National Research Foundation of Korea (NRF-2009-0090559). The work at KIST was supported by the KIST Institutional Program and the Korea-Sweden Research Cooperation Program.

${ }^{1}$ M. Tonouchi, Nat. Photonics 1, 97 (2007).

${ }^{2}$ K. Sakai, TerahertzOptoelectronics (Springer, Berlin, 2005).

${ }^{3}$ X.-C.Zhang, J. T. Darrow, B. B. Hu, D. H. Auston, M. T. Schmidt, P. Tham, and E. S. Yang, Appl. Phys. Lett. 56, 2228 (1990).

${ }^{4}$ R. Ascazubi, I. Wilke, K. Denniston, H. Lu, and W. J. Schaff, Appl. Phys. Lett. 84, 4810 (2004).

${ }^{5}$ S. L. Chuang, S. Schmitt-Rink, B. I. Greene, P. N. Saeta, and A. F. J. Levi, Phys. Rev. Lett. 68, 102 (1992).

${ }^{6}$ P. Gu, M. Tani, S. Kono, K. Sakai, and X.-C. Zhang, J. Appl. Phys. 91, 5533 (2002).

${ }^{7}$ A. Nainani, D. Kim, T. Krishnamohan, K. Saraswat, "Hole Mobility and its Enhancement with Strain for Technologically Relevant III-V Semiconductors," Proceedings of SISPAD 2009, pp. 47-50.

${ }^{8}$ T. Dekorsy, H. Auer, H. J. Bakker, H. G. Roskos, and H. Kurz, Phys. Rev. B 53, 4005 (1996).

${ }^{9}$ M. Yi, K. Lee, J. Lim, Y. Hong, Y. D. Jho, and J. Ahn, Opt. Express 18, 13693 (2010).

${ }^{10}$ G. Klatt, B. Surrer, D. Stephan, O. Schubert, M. Fischer, J. Faist, A. Leitenstorfer, R. Huber, and T. Dekorsy, Appl. Phys. Lett. 98, 021114 (2004).

${ }^{11}$ R. McLaughlin, A. Corchia, M.B. Johnston, Q. Chen, C.M. Ciesla, D.D. Arnone, G.A.C. Jones, E.H. Linfield, A.G. Davies, and M. Pepper, Appl. Phys. Lett. 76, 2038 (2000).

${ }^{12}$ A. Dreyhaupt, S. Winnerl, T. Dekorsy, and M. Helm, Appl. Phys. Lett. 86, 121114 (2005).

${ }^{13}$ D. Grischkowsky, S. Keiding, M. van Exter, and C. Fattinger, J. Opt. Soc. Am. B, 7, 2006 (1990).

${ }^{14}$ M. Reid and R. Fedosejevs, Appl. Phys. Lett. 86, 011906 (2005).

${ }^{15}$ K. Liu, J. Xu, T. Yuan and X.-C. Zhang, Phys. Rev. B 73, 155330 (2006).

${ }^{16}$ H. Jeong, S.H. Shin, S.Y. Kim, J.D. Song, S.B. Choi, D.S. Lee, J. Lee, and Y.D. Jho, Curr. Appl. Phys. 12, 668-672 (2012).

${ }^{17}$ H. Fu, L.-W. Wang, and A. Zunger, Phys. Rev. B 59, 5568 (1999).

${ }^{18}$ C. T. Que, T. Edamura, M. Nakajima, M. Tani, and M. Hangyo, 48, 010211 (2009).

${ }^{19}$ M.B. Johnston, D.M. Whittaker, A. Corchia, A.G. Davies, and E.H. Linfield, Phys. Rev. B, 65, 165301 (2002). 